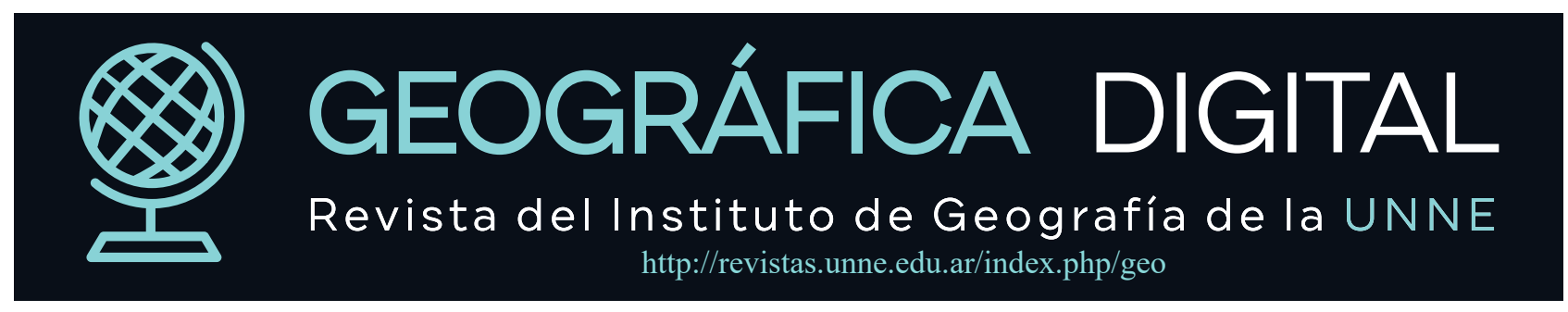

Revista Geográfica Digital, 2020, Vol. 17, No 34, 92-107 pp., E-ISSN: 1668-5180: DOI: http://dx.doi.org/10.30972/geo.17344525

\title{
Elementos metodológicos para optimizar la gestión del cambio climático a nivel local en Colombia
}

\section{Methodological elements to optimize climate change management at local level in Colombia}

\section{Rodriguez Caicedo, Jeniffer A. ${ }^{\text {; }}$ Vásquez Lizcano, Jonathan ${ }^{2}$}

${ }^{1}$ Facultad de Ciencias Ambientales y de la Sostenibilidad. Universidad de Ciencias Aplicadas y Ambientales (U.D.C.A). jenirodriguez@udca.edu.co ${ }^{2}$ Facultad de Ciencias Ambientales y de la Sostenibilidad. Universidad de Ciencias Aplicadas y Ambientales (U.D.C.A). jlizcanova@gmail.com

Palabras Claves:

Gestión del cambio climático local,

Ley 1931 de 2018

Política Nacional de Cambio Climático

Ordenamiento territorial Elementos metodológicos

\section{Keywords:}

Local climate change management

Law 1931 of 2018

National Policy on Climate

Change

Land use planning

Elements

\section{R E S U M E N}

El objetivo del presente trabajo consistió en diseñar una guía con elementos metodológicos principales para optimizar la gestión del cambio climático a nivel local en Colombia, para lo cual se tuvo en cuenta en principio lo establecido en el marco normativo internacional, así como la normatividad en Colombia: Ley 1931 del 2018; Política Nacional de Cambio Climático y documentos con consideraciones de cambio climático para el ordenamiento territorial. Así como la revisión y comparación de herramientas e instrumentos locales de cuatro países en Latinoamérica: Chile, Paraguay, Costa Rica y Brasil. Así mismo, en el ámbito nacional se tomaron como referencia dos departamentos: Risaralda y Huila; y tres municipios: Montería, Tuluá y Topagá. Finalmente se propone una guía de elementos metodológicos para optimizar la gestión del cambio climático a nivel local en Colombia teniendo en cuenta consideraciones desde el ordenamiento territorial a través de una hoja de ruta que permita a los encargados locales optimizar la gestión del cambio climático.

\section{A B S T R A C T}

The objective of this work consisted in designing a guide to optimize the management of climate change at local level in Colombia. For this, the provisions of the international regulatory framework were taken into account; as well as the national regulations in Colombia: Law 1931 of 2018; National Policy on Climate Change; and documents with climate change considerations for land use planning. This included the review and comparison of local tools and instruments from four countries in Latin America: Chile, Paraguay, Costa Rica and Brazil, likewise at national level two departments were taken as a reference: Risaralda and Huila; and three municipalities: Montería, Tuluá and Topagá. Guide design took into account considerations from land use policy through instructions that allow local managers to optimize the management of climate change. 


\section{Introducción}

En el desarrollo de este trabajo se asumió el cambio climático como lo define el Panel Intergubernamental de Cambio Climático (IPCC) como la variación del estado del clima identificable en las variaciones del valor medio y/o en la variabilidad de sus propiedades, que persiste durante largos períodos de tiempo, generalmente decenios o períodos más largos. El cambio climático puede deberse a procesos internos naturales o a forzamientos externos tales como modulaciones de los ciclos solares, erupciones volcánicas o cambios antropógenos persistentes de la composición de la atmósfera o del uso del suelo (Grupo Intergubernamental de Expertos sobre Cambio Climático (IPCC), 2013). Así mismo, se toma la definición de gestión del cambio climático establecida en la Política Nacional de Cambio Climático de la República de Colombia (PNCC), definiéndose como el proceso coordinado de diseño, implementación y evaluación de acciones de mitigación de Gases de Efecto Invernadero (GEI) y adaptación al cambio climático, orientado a reducir la vulnerabilidad de la población, infraestructura y ecosistemas a los efectos del cambio climático, así como la inclusión de las acciones orientadas a permitir y aprovechar las oportunidades que el cambio climático genera (Ministerio de Medio Ambiente y Desarrollo Sostenible, 2017). Valga la pena resaltar la diferencia entre "gestión del cambio climático" y "adaptación al cambio climático", pues el primero es un concepto más amplio que engloba al segundo; la gestión incluye no solamente procesos de adaptación sino de mitigación, entre otros.

Desde la naturaleza de los riesgos asociados al cambio climático, la Ley 1931 de 2018 plantea la importancia de adoptar una visión territorial, que valore e incorpore articuladamente iniciativas para lograr una gestión del cambio climático acertada y efectiva. Desde el documento del Consejo Nacional de Política Económica y Social (CONPES) 3700, documento que plantea la estrategia institucional para la articulación de políticas y acciones en materia de cambio climático en Colombia, que responde al Plan Nacional de Adaptación al Cambio Climático, se propuso establecer un marco de coordinación por medio del cual los sectores, los territorios y las comunidades siguieran una ruta para entender el cambio climático como un asunto de desarrollo económico y social, y así mismo integrarlo dentro de sus procesos de planificación e inversión (Departamento Nacional de Planeación de Colombia, 2011).

Para la integración del cambio climático en los procesos de planificación el CONPES 3700 propuso la 'Hoja de ruta para la elaboración de los planes de adaptación dentro del Plan Nacional de Adaptación al Cambio Climático' como guía dirigida a los responsables en la toma de decisiones, con el propósito de mejorar los procesos que integran la adaptación al cambio climático a nivel local, identificando las modificaciones y la construcción de insumos requeridos para incorporar en otros instrumentos de planificación, teniendo en cuenta los potenciales efectos del cambio climático como criterios de decisión.

Siguiendo lo establecido en el documento CONPES 3700, el Ministerio de Medio Ambiente y Desarrollo Sostenible (MADS) planteó la Política Nacional de Cambio Climático (PNCC), que estableció estrategias territoriales de gestión del cambio climático desde la adaptación y la mitigación de gases de efecto invernadero (GEI) y lineamientos para su articulación buscando la optimización de sinergias entre distintos criterios y elementos del territorio, necesarios para relacionar la adaptación y la mitigación de GEI con decisiones relevantes de desarrollo. Una de las estrategias territoriales que se estableció es la formulación de planes integrales de gestión del cambio climático territoriales para períodos de 12 años (Ministerio de Medio Ambiente y Desarrollo Sostenible de Colombia, 2017); sin embargo, para la formulación de estos planes aún no se han establecido los lineamientos con miras al proceso de gestión del cambio climático a nivel local, lo cual implica hacer énfasis en los procesos que afectan a una comunidad y no tanto a otras (Christlieb, 2012), desde sus bases conceptuales.

Actualmente Colombia solo cuenta con 20 planes referentes al cambio climático, de los cuales 16 responden a un carácter integral (Ministerio de Medio Ambiente y Desarrollo Sostenible de Colombia, 2020) y a escala municipal, de 1103 municipios solo seis cuentan con un plan referente al cambio climático y solamente uno con carácter integral. Una de las posibles causas del por qué los municipios no han construido un plan hacia la gestión de cambio climático, es atribuido a que dentro del CONPES 3700 se estableció una guía conceptual enfocada a la adaptación de cambio climático nombrada 'Hoja de ruta para la elaboración de los planes de adaptación dentro del Plan Nacional de Adaptación al Cambio Climático', y estos pocos planes formulados son un intento de integración de criterios territoriales enmarcados hacia la adaptación más no el proceso de gestión del cambio climático.

Por tal razón, este trabajo tuvo como finalidad identificar los elementos metodológicos principales necesarios para optimizar la gestión del cambio climático a nivel local en Colombia desde la descripción de herramientas e instrumentos internacionales y nacionales asociados a la gestión del cambio climático. En el ámbito internacional se revisaron las Políticas Nacionales referentes al cambio climático de cuatro países: República de Chile, Republica de Paraguay, Republica de Costa Rica y República Federativa de Brasil; 
luego, se documentaron las herramientas que actúan como hojas de rutas para los responsables en la toma de decisiones y, finalmente, un instrumento local de planificación referente al cambio climático. En el ámbito nacional se revisaron los instrumentos de planificación referentes a la gestión del cambio climático de dos departamentos: Risaralda y Huila, y tres municipios: Montería, Tuluá y Topagá.

Finalmente, derivando en una guía que permita establecer los elementos metodológicos principales para la gestión del cambio climático a nivel local con un carácter integral, teniendo en cuenta el marco normativo nacional e internacional aplicable para Colombia, así como algunas consideraciones de documentos investigados, lo que generará una optimización de la gestión del cambio climático a nivel local en Colombia.

\section{Antecedentes}

En un contexto general, el concepto de gestión de cambio climático es poco referenciado en su forma literal; sin embargo, hay documentos oficiales tanto en el ámbito internacional como en el local que han aportado a la construcción del concepto. Un primer acercamiento se dio en la Convención Marco de las Naciones Unidas sobre el Cambio Climático (CMNUCC), en la cual se partió de la necesidad de coordinar las acciones para hacer frente al aumento de las emisiones de gases de efecto invernadero (GEI), así como el planteamiento de medidas para contrarrestar sus impactos sobre la población y actividades humanas (CMNUCC, 1997).

En el 2002, en Colombia se publicó la primera comunicación nacional ante la CMNUCC, coordinada por el Instituto de Hidrología, Meteorología y Estudios Ambientales (IDEAM), el entonces Ministerio de Medio Ambiente y con apoyo del Departamento Nacional de Planeación (DNP), estos elaboraron el documento: 'lineamientos de Política de Cambio Climático' con el fin de identificar estrategias para la consolidación de la capacidad nacional necesaria que permita responder a las posibles amenazas del cambio climático; y que responde a las disposiciones de la convención, en términos de potencializar oportunidades derivadas de los mecanismos financieros y cumplir con los compromisos establecidos (Departamento Nacional de Planeación; Ministerio de Medio Ambiente y Desarrollo Sostenible; Instituto de Hidrología y Meteorología, 2002).

En 2010, el país comunicó sus compromisos preliminares nacionales, en términos de medidas apropiadas de mitigación y a través del IDEAM remitió la segunda comunicación nacional, en la que se presentó la evolución de las políticas y acciones colombianas. A partir de allí, se comenzaron a trabajar varias estrategias para abordar la problemática del cambio climático, plasmadas en el Plan Nacional de desarrollo 2010 - 2014 'Prosperidad para Todos'.

La estrategia que se considera más relevante con relación a la gestión del cambio climático es el PNACC; en este plan se consolidó un marco conceptual para la adaptación al cambio climático en el país y se establecieron los lineamientos que se deberán seguir durante el proceso de formulación de los planes sectoriales y territoriales de adaptación. Es aquí donde se empezó a considerar que la gestión del cambio climático debe ser incorporada en los diferentes sectores del desarrollo, desde la fase de planeación a nivel local, integrando las variables climáticas en los Planes de Desarrollo, así como en los Planes de Ordenamiento Territorial (POT) y los Planes de Ordenamiento y Manejo de Cuencas Hidrográficas (POMCA) (Programa de las Naciones Unidas para el Desarrollo; Ministerio de Ambiente y Desarrollo Sostenible; Instituto de Hidrología y Meteorología, 2010).

Posteriormente, en el 2011 se expidió el documento CONPES 3700 del 2011 que entre tantos objetivos propuso:

un marco de coordinación por medio del cual, los sectores, los territorios y las comunidades, entendieran el cambio climático como un asunto de desarrollo económico y social, $y$ por tanto integraran dicha problemática dentro de sus procesos de planificación; y un marco de coordinación adecuado para que pudieran ser implementadas las acciones priorizadas en su momento por el país (Departamento Nacional de Planeación, 2011, p.35).

Es en este marco de coordinación, se mencionó por primera vez a los nodos regionales de cambio climático, como una medida de descentralización de las acciones nacionales, en la búsqueda del empoderamiento de los entes territoriales y de las poblaciones locales frente a la gestión para enfrentar el cambio climático en Colombia (Ministerio de Medio Ambiente y Desarrollo Sostenible de Colombia, 2016).

En el Plan Nacional de Desarrollo 2014-2018 'Todos por un nuevo país', se instauraron estrategias de crecimiento verde definido como un enfoque que propende por un desarrollo sostenible que garantice 
el bienestar económico y social de la población en el largo plazo, asegurando que la base de los recursos provea los bienes y servicios ecosistémicos que el país necesita y el ambiente natural sea capaz de recuperarse ante los impactos de las actividades productivas, temas asociados al cambio climático y se hizo explícita la necesidad de consolidar la política nacional de cambio climático, buscando su integración con la planificación ambiental, territorial y sectorial. A raíz de esto, el actual Ministerio de Medio Ambiente y Desarrollo Sostenible (MADS) junto al Programa de las Naciones Unidas para el Desarrollo (PNUD), desarrolló el documento 'Consideraciones de cambio climático para el ordenamiento territorial', con el fin de ofrecer consideraciones de cambio climático, para tener en cuenta a la hora de revisar y ajustar los planes de ordenamiento territorial, planteando una visión de modelo de desarrollo territorial baja en carbono y resiliente al clima (Programa de las Naciones Unidas para el Desarrollo; Ministerio de Ambiente y Desarrollo Sostenible; Gobierno de Colombia, 2018).

En el 2018, el Programa de las Naciones Unidas para el Desarrollo (PNUD) junto al MADS, elaboraron el documento 'Consideraciones de cambio climático para el ordenamiento territorial', dirigido a los municipios y distritos, para que en sus procesos de ordenamiento territorial, consideren las condiciones actuales y futuras del clima, ya que esto condiciona su desarrollo y crecimiento; así mismo, estableció los instrumentos de ordenamiento territorial como herramientas útiles para gestionar las manifestaciones del cambio climático, y promover un desarrollo bajo en carbono y resiliente al clima, desde ocho insumos claves para sus etapas de diagnóstico y formulación: estructura ecológica, sistemas productivos, equipamientos colectivos, espacio público, vivienda y hábitat, movilidad, vías y transportes, patrimonio cultural y servicios públicos domiciliarios. Este documento incluyó anexos técnicos y metodológicos para facilitar la comprensión de los elementos útiles para implementar acciones frente al cambio climático que tienen incidencia directa en la ocupación o el desarrollo territorial (Programa de las Naciones Unidas para el Desarrollo; Ministerio de Ambiente y Desarrollo Sostenible; Gobierno de Colombia, 2018).

Y finalmente, para consolidar los esfuerzos en materia de gestión de cambio climático en el país, se promulgó la Ley 1931 del 27 de Julio de 2018, la cual estableció directrices para la gestión del cambio climático, definiéndolo como el "proceso coordinado de diseño, implementación y evaluación de acciones de mitigación de Gases de Efecto Invernadero y adaptación al cambio climático orientado a reducir la vulnerabilidad de la población, infraestructura y ecosistemas a los efectos del cambio climático"; así mismo, estableció que las autoridades municipales y distritales deberán incorporar dentro de sus planes de desarrollo y planes de ordenamiento territorial, la gestión del cambio climático teniendo como referencia los Planes Integrales de Gestión del Cambio Climático Territoriales de su departamento y los Planes Integrales de Gestión del Cambio Climático Sectoriales (Congreso de la República de Colombia, 2018).

\section{Materiales y métodos}

Para la elaboración de la guía, primero se hizo una revisión bibliográfica en el ámbito internacional y nacional de instrumentos y herramientas referentes a la gestión del cambio climático a nivel local. Internacionalmente se indagó en cuatro países de Latinoamérica: Chile, Costa Rica, Brasil y Paraguay, y nacionalmente en dos departamentos: Huila y Risaralda, y tres municipios: Montería en el departamento de Córdoba, Tuluá en el departamento de Valle del Cauca y Topagá en el departamento de Boyacá.

Los documentos a nivel nacional consultados fueron: 'Consideraciones de cambio climático para el ordenamiento territorial' elaborado por el MADS (Programa de las Naciones Unidas para el Desarrollo; Ministerio de Ambiente y Desarrollo Sostenible; Gobierno de Colombia, 2018), que tiene como fin contribuir a la construcción de instrumentos de ordenamiento territorial más estratégicos, más innovadores y resilientes al clima; otro documento fue 'Orientaciones para la inclusión del cambio climático en los planes de ordenamiento territorial (Planes de Ordenamiento Territorial (POT), Planes Básicos de Ordenamiento Territorial (PBOT), Esquemas de Ordenamiento Territorial (EOT))' (Corporacion Autónoma Regional de Colombia, 2018), que se fundamenta en las directrices nacionales dadas por el MADS y otros referentes que han aportado significativamente en la gestión del cambio climático y en el ejercicio del ordenamiento territorial a nivel nacional.

Desde el documento 'Consideraciones de cambio climático para el ordenamiento territorial' (Programa de las Naciones Unidas para el Desarrollo; Ministerio de Ambiente y Desarrollo Sostenible; Gobierno de Colombia, 2018) se tuvo en cuenta la dinámica territorial y el cambio climático que desde el artículo segundo de la Ley Orgánica de Ordenamiento Territorial define el instrumento de ordenamiento territorial como una manera de organización político-administrativa de los territorios, teniendo como propósitos: promover el desarrollo institucional y fortalecer la identidad cultural y el desarrollo territorial atendiendo a la diversidad cultural y físico-geográfica de Colombia, por lo cual se realizaron algunas matrices donde se consignaron los roles de los gestores de cambio climático, se realizó una revisión 
demográfica de minorías étnicas y las características físicas del territorio que se vaya a gestionar.

Por lo anterior, fue preciso hacer una revisión de los instrumentos de ordenamiento como herramientas para considerar acciones de cambio climático sobre el territorio y, en especial, sobre los sistemas que lo estructuran, entre los que se encuentran: estructura ecológica principal, sistemas productivos, equipamientos colectivos, espacio público, vivienda y hábitat, movilidad, vías y transporte, el patrimonio cultural, y servicios públicos domiciliarios (Figura 1).

Figura 1. Convergencia de la gestión del cambio climático y la planificación territorial municipal en los sistemas estructurantes.
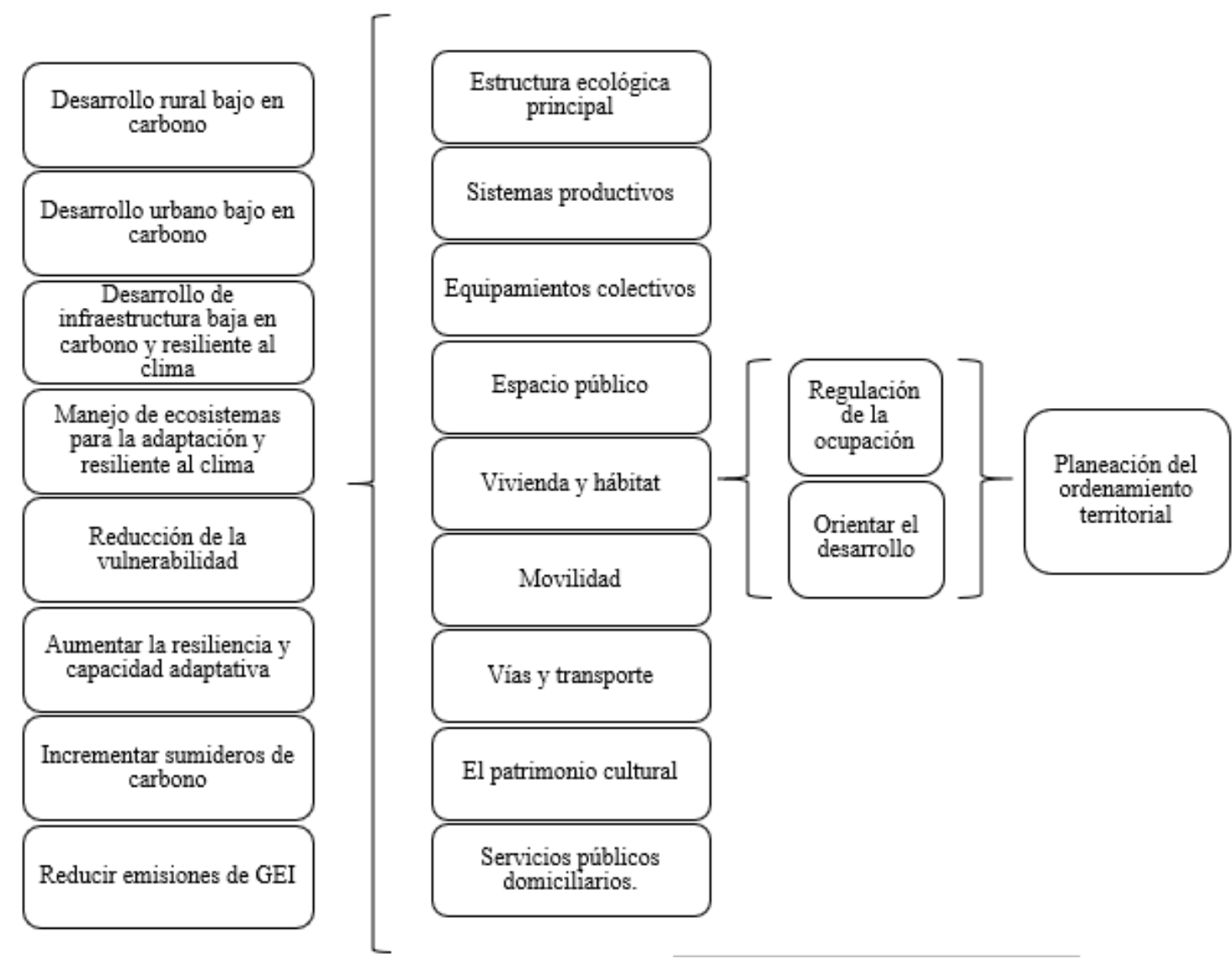

Fuente: adaptado del Programa de las Naciones Unidas para el Desarrollo; Ministerio de Ambiente y Desarrollo Sostenible, 2018.

Así mismo, desde el documento 'Orientaciones para la inclusión del cambio climático en los planes de ordenamiento territorial (POT, PBOT, EOT)' (Corporacion Autónoma Regional, 2018) se tuvieron en cuenta las clasificaciones del suelo: urbano, suburbano y rural; para que desde estos se localicen los riesgos según el suelo, se identifiquen las normativas propias de cada uno y se formulen estrategias más eficaces para la gestión del cambio climático en el territorio.

Finalmente se tuvo en cuenta un lineamiento referente al seguimiento de la gestión, pues los instrumentos de planificación pueden actualizarse junto a sus estrategias y medios de implementación, así como los estudios científicos en materia de cambio climático, pues este fenómeno puede variar y las medidas que se establezcan deben ir en concordancia con la realidad climática y territorial.

\section{Resultados}

Se diseñó una guía en la cual se establecieron los elementos metodológicos principales que contribuyen a optimizar la gestión del cambio climático en Colombia a nivel local respondiendo al carácter integral de la gestión, lo que implica planificar acciones abordando el cambio climático desde la agenda de desarrollo, que para el Estado colombiano el principal enfoque se traduce en crecimiento económico dejando de lado el enfoque ambiental. En este orden de ideas se realizó un inventario de la información que se conoce en relación con el clima, sus impactos, actores involucrados, identificando las acciones propuestas en otros instrumentos de planificación, permitiendo orientar a los tomadores de decisiones locales, en el uso y manejo del territorio con miras a afrontar los efectos del cambio y la variabilidad climática en un horizonte de 30 años. 
Para optimizar la gestión del cambio climático a nivel local en Colombia, se proponen cuatro elementos metodológicos principales (Figura 2): planificación y diagnóstico, formulación de estrategias, medios de implementación y seguimiento de la gestión, estos corresponden a cuatro etapas para planificar la gestión de cambio climático en un horizonte de 30 años: En la primera etapa se llevará a cabo la identificación de la gestión del cambio climático, la recolección de información física y socioeconómica, un perfil de gestión climático local, y el análisis de riesgos climáticos locales del territorio; en la segunda etapa se formularán y concretarán las estrategias de gestión que aumenten la resiliencia del territorio ante los riesgos identificados; en la tercera etapa se definirán los medios de implementación para la ejecución de las estrategias que se plantearon en la etapa anterior, identificando los actores y el rol que cumplirán; y, finalmente, en la cuarta etapa se proponen herramientas de seguimiento anual para la gestión del cambio climático a nivel local.

Figura 2. Etapas de la guía con elementos metodológicos principales para optimizar la gestión del cambio climático a nivel local en Colombia.

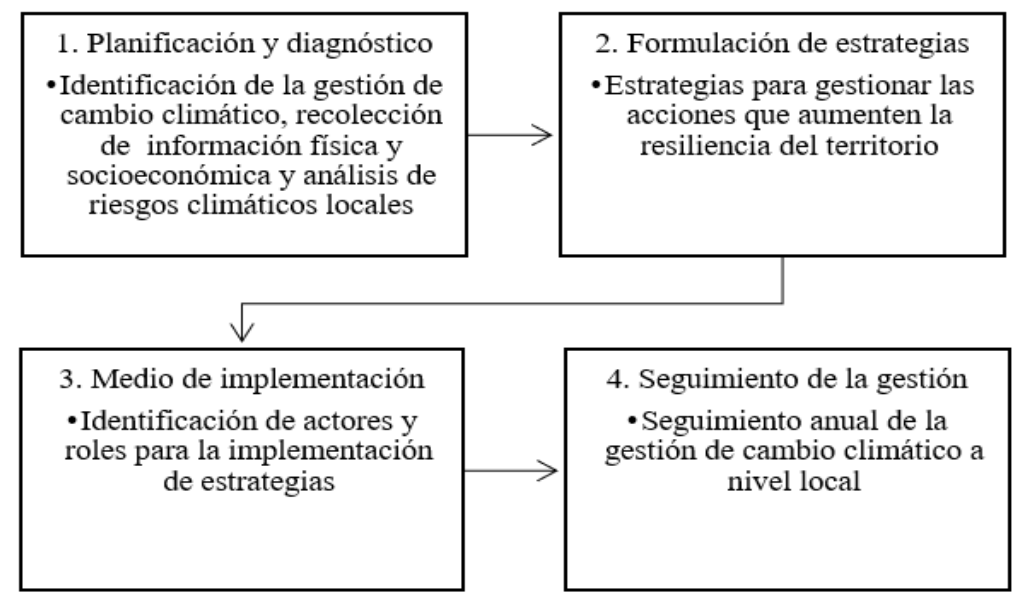

Fuente: elaboración propia.

\subsection{Etapa 1: Planificación y diagnóstico}

La etapa de planificación y diagnóstico de esta guía consiste en reconocer el territorio desde la gestión del cambio climático y del territorio, a través de la identificación de acciones en otros instrumentos de planificación; la información física y sociodemográfica local, un perfil de gestión climática local y análisis de los riesgos climáticos locales (Figura 3). Los aspectos físicos y socioeconómicos permiten delimitar el territorio e identificar la población del territorio, el perfil climático local reúne la información que permite entender los impactos del cambio climático en la realidad local, y el análisis de vulnerabilidad climática local permite identificar debilidades y fortalezas para la adaptación al cambio climático, permitiendo ver los impactos en el territorio.

Figura 3. Metodología de la etapa diagnóstico.

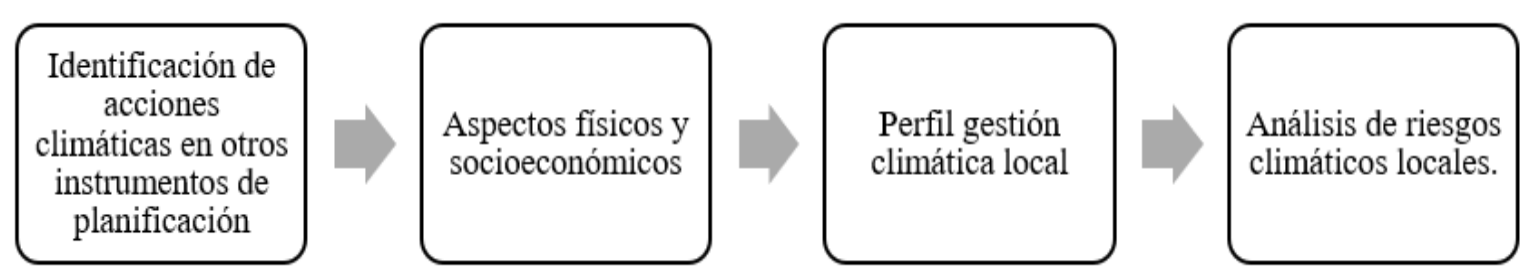

Fuente: elaboración propia.

\subsubsection{Identificación de la gestión de cambio climático en otros instrumentos de planificación territorial}

En esta sección se identificarán las consideraciones de cambio climático en otros instrumentos de planificación, como lo son Planes de Ordenamiento Territorial (POT), Esquemas de Ordenamiento Territorial (EOT), Planes Básicos de Ordenamiento Territorial (PBOT), Planes de Ordenamiento y Manejo 
de Cuencas (POMCA), Planes de Ordenamiento y Manejo de Unidad Ambiental Costera (POMIUAC), entre otros. Para esto se propone la siguiente matriz (Tabla 1) que permite recopilar y organizar la información de cambio climático en los instrumentos territoriales locales del territorio que se esté gestionando, indicando horizonte de planificación, nombre de los programas y/o proyectos en el componente programático de los instrumentos referentes al cambio climático y las fuentes de financiación:

Tabla1. Matriz identificación de cambio climático en otros instrumentos de planificación territorial.

\begin{tabular}{|c|c|c|c|}
\hline \multirow{2}{*}{$\begin{array}{l}\text { Instrumento } \\
\text { de } \\
\text { planificación }\end{array}$} & \multirow[b]{2}{*}{$\begin{array}{l}\text { Horizonte de } \\
\text { planificación }\end{array}$} & \multicolumn{2}{|c|}{$\begin{array}{c}\text { Componente programático } \\
\text { de cambio climático }\end{array}$} \\
\hline & & $\begin{array}{c}\text { Nombre } \\
\text { programas } \\
\text { y/o proyectos }\end{array}$ & $\begin{array}{l}\text { Fuentes de } \\
\text { financión }\end{array}$ \\
\hline $\begin{array}{l}\text { POT, EOT, } \\
\text { PBOT, } \\
\text { POMCA, } \\
\text { POMIUAC, } \\
\text { entre otros }\end{array}$ & 12 años & $\begin{array}{l}\text { Programa } \\
\text { acción por el } \\
\text { clima }\end{array}$ & $\$ 12.000 .000$ \\
\hline
\end{tabular}

Fuente: elaboración propia.

Posteriormente se propone una matriz para identificar los datos del Plan de Desarrollo (PD) en curso, sobre los programas y proyectos relativos al cambio climático, tomando como base la sección del Plan Plurianual de Inversiones (PPI), que documenta el presupuesto en el cuatrienio del PD. En la Tabla 2 se presenta un formato de matriz que puede ser aplicado para la identificación de acciones de cambio climático en el PPI, donde se encuentra el sector de desarrollo, que corresponde a las clasificaciones que se usen para la formulación de los programas y proyectos, como por ejemplo, medio ambiente, agua potable y saneamiento básico entre otros; el nombre del programa o proyecto; la inversión en pesos y su porcentaje para el cuatrienio.

Tabla 2. Identificación de la inversión para cambio climático en el PPI del PD.

\begin{tabular}{|c|c|c|c|}
\hline $\begin{array}{l}\text { Sector de } \\
\text { desarrollo }\end{array}$ & $\begin{array}{c}\text { Programas y/o } \\
\text { proyectos }\end{array}$ & $\begin{array}{l}\text { Inversión } \\
\text { PPI }\end{array}$ & $\begin{array}{c}\text { Inversión } \\
\text { PPI (\%) }\end{array}$ \\
\hline $\begin{array}{l}\text { Cambio } \\
\text { climático }\end{array}$ & Acción por el clima & $\$ 3.000 .000$ & $2 \%$ \\
\hline \multicolumn{2}{|r|}{ Subtotal } & $\$ 3.000 .000$ & $2 \%$ \\
\hline \multicolumn{2}{|r|}{ Total } & $\$ 3.000 .000$ & $2 \%$ \\
\hline
\end{tabular}

Fuente: elaboración propia.

Se identificarán los grupos sociales, gremios, organizaciones, líderes locales e industrias, entre otros actores, que intervienen en el desarrollo del municipio; posteriormente se asignarán las funciones y deberes en los que interviene la comunidad que contribuyan a la optimización de la gestión del cambio climático, pues son ellos quienes conocen de primera mano la tendencia climatológica del municipio (Tabla 3).

Tabla 3. Actores involucrados en la gestión del cambio climático.

\begin{tabular}{|c|c|c|c|c|}
\hline $\begin{array}{c}\text { Nombre } \\
\text { del actor } \\
\text { (persona, } \\
\text { organización, } \\
\text { entidad) }\end{array}$ & $\begin{array}{c}\text { Gubernamental/ No } \\
\text { gubernamental }\end{array}$ & Función (es) & Fortalezas & Debilidades \\
\hline $\begin{array}{c}\text { Corporación } \\
\text { Autónoma } \\
\text { Regional } \\
\text { (CAR) }\end{array}$ & Gubernamental & $\begin{array}{c}\text { Capacitación } \\
\text { técnica }\end{array}$ & $\begin{array}{c}\text { Relaciones } \\
\text { supramunicipales } \\
\text { en materia de } \\
\text { cambio climático }\end{array}$ & $\begin{array}{c}\text { Funciones regionalizadas } \\
\text { y no locales }\end{array}$ \\
\hline
\end{tabular}

Fuente: elaboración propia.

Finalmente, se identificarán las autoridades en materia de gestión de cambio climático, como lo son el nodo regional de cambio climático y la corporación autónoma regional; además, se representará cartográficamente la división político-administrativa y la zonificación hidrográfica (Figura 4). 
Figura 4. Identificación cartográfica de autoridades en materia de gestión de cambio climático.

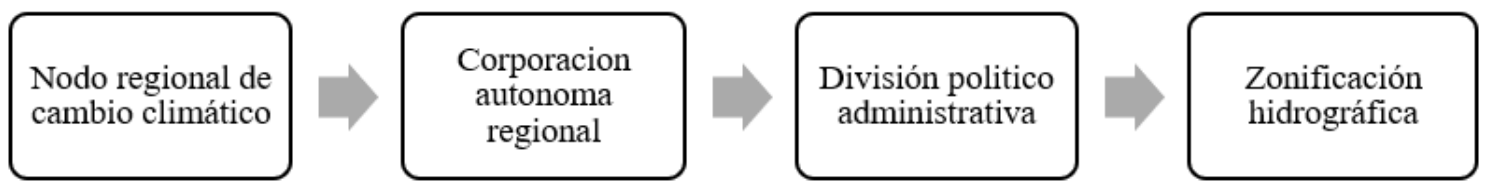

Fuente: elaboración propia.

\subsubsection{Información física y socioeconómicos}

Para la información física se definirá la delimitación geográfica del territorio, una descripción general desde los aspectos físicos como lo son: la hidrología, el clima, la geología, los suelos, la vegetación y/o actividades agrícolas, entre otros, que permitan reconocer el territorio para el cual se realizará la gestión de cambio climático. Así como la producción cartográfica de estos aspectos, y la georreferenciación de los riesgos climáticos locales correspondientes al territorio que se está gestionando, estos productos cartográficos se tendrán en cuenta para la sección de análisis de riesgos climáticos locales.

Para identificar los aspectos socioeconómicos (Tabla 4), se propone organizar los datos poblacionales, como lo es la población actual, proyección en 20 años de esta población, densidad poblacional, la identificación de minorías étnicas, la clasificación económica del municipio y las principales actividades económicas, entre otros, que considere pertinente adicionar el gestor.

Tabla 4. Aspectos socioeconómicos del municipio.

\begin{tabular}{|c|c|c|}
\hline $\begin{array}{l}\text { Población actual } \\
\text { (Número de } \\
\text { habitantes) }\end{array}$ & \multicolumn{2}{|c|}{10.000} \\
\hline $\begin{array}{c}\text { Proyección } \\
\text { población en } 20 \\
\text { años (Número de }\end{array}$ & \multicolumn{2}{|c|}{20.000} \\
\hline \multirow{4}{*}{$\begin{array}{l}\text { Minoría étnicas } \\
\text { (Número de } \\
\text { habitantes) }\end{array}$} & Indígena & 300 \\
\hline & ROM (Gitana) & 35 \\
\hline & $\begin{array}{c}\text { Raizal del } \\
\text { archipiélago de } \\
\text { San Andrés y } \\
\text { Providencia }\end{array}$ & 0 \\
\hline & $\begin{array}{l}\text { Negro, mulato, } \\
\text { afrocolombiano o } \\
\text { afrodescendiente }\end{array}$ & 140 \\
\hline $\begin{array}{l}\text { Clasificación } \\
\text { económica del } \\
\text { municipio }\end{array}$ & \multicolumn{2}{|c|}{ Sexta categoría } \\
\hline $\begin{array}{l}\text { Principales } \\
\text { actividades } \\
\text { económicas }\end{array}$ & \multicolumn{2}{|c|}{$\begin{array}{c}\text { Minería } \\
\text { Agroindustria }\end{array}$} \\
\hline
\end{tabular}

Fuente: elaboración propia.

\subsubsection{Perfil de gestión climática local}

El perfil de gestión climática local (Tabla 5) permite conocer el estado de la gestión en el territorio. Se diligenciarán las autoridades en materia de gestión de cambio climático identificadas anteriormente; así mismo se tendrán en cuenta los planes programas y proyectos identificados en los instrumentos de planificación; también la capacidad humana encargada o relacionada con la gestión de cambio climático a nivel local; el presupuesto con el que cuentan y si los funcionarios están capacitados en materia de cambio climático. 
Tabla 5. Perfil de gestión climática local.

\begin{tabular}{|c|c|}
\hline Nodo regional de cambio climático: & $\begin{array}{l}\text { A6 Región } \\
\text { Andina }\end{array}$ \\
\hline Corporación autónoma regional & CAR \\
\hline Zonificación hidrográfica & $\begin{array}{l}\text { 2401 Río } \\
\text { Suárez }\end{array}$ \\
\hline $\begin{array}{c}\text { № de funcionarios encargados de tratar } \\
\text { temas ambientales }\end{array}$ & 4 \\
\hline $\begin{array}{c}\text { № de funcionarios capacitados en temas } \\
\text { ambientales }\end{array}$ & 4 \\
\hline $\begin{array}{l}\text { ¿Cuenta el municipio con unidad de } \\
\text { bomberos? }\end{array}$ & No \\
\hline $\begin{array}{l}\text { № de funcionarios encargados de la unidad } \\
\text { de bomberos }\end{array}$ & 0 \\
\hline $\begin{array}{l}\text { Presupuesto total asignado a la unidad en } \\
\text { año actual }\end{array}$ & 0 \\
\hline $\begin{array}{l}\text { № de funcionarios capacitados en temas de } \\
\text { cambio climático }\end{array}$ & 1 \\
\hline
\end{tabular}

Fuente: matriz adaptada de Municipalidad de Vitacura, 2019.

\subsubsection{Análisis de riesgos climáticos locales}

En este apartado, el gestor de cambio climático local analizará los riesgos climáticos identificados en los productos cartográficos realizados en la sección 4.1.2. El análisis se realizará desde la evaluación de la amenaza y vulnerabilidad del territorio, indicando el nivel de riesgo en los sistemas estructurantes del territorio que se ven afectados con la ocurrencia de estos riesgos.

Los sistemas estructurantes del territorio son siete: movilidad, vías y transporte, equipamientos colectivos, espacio público, sistemas productivos, estructura ecológica principal, servicios públicos domiciliarios, vivienda y hábitat y patrimonio cultural. La amenaza será evaluada mediante cuatro factores: frecuencia, duración, probabilidad y consecuencia; asimismo, para la vulnerabilidad se tendrán en cuenta tres factores: exposición, fragilidad y resiliencia, para finalmente poder determinar el nivel de riesgo (amenaza + vulnerabilidad) que las amenazas representan para cada sistema afectado. A continuación, se presentan los criterios para estas evaluaciones y la determinación del nivel de riesgo.

\subsubsection{Criterios de evaluación de amenazas locales}

Para la evaluación de amenazas se partirá de las potenciales ocurrencias de riesgos climáticos que pueden tener un impacto físico, social, económico y ambiental en el territorio; los criterios que se tendrán en cuenta son: la frecuencia que se conocerá como el nivel de ocurrencia en que se repite el evento climático, la duración que es el periodo de tiempo en que la población está expuesta al evento climático, la probabilidad de que ocurra el evento y la consecuencia que hace referencia a los daños generados por el evento en la vida humana, ecosistemas y servicios ambientales. En la Tabla 6 se presenta la matriz con los criterios de evaluación de la amenaza.

Tabla 6. Criterios de evaluación de amenazas locales para la gestión de cambio climático.

\begin{tabular}{|c|c|c|c|c|}
\hline $\begin{array}{l}\text { Rangos de } \\
\text { evaluación }\end{array}$ & Frecuencia & Duración & Probabilidad & Consecuencia \\
\hline $\begin{array}{l}\text { Muy alto ( } 5 \\
\text { puntos) }\end{array}$ & $\begin{array}{l}\text { Varias veces al } \\
\text { año }\end{array}$ & $\begin{array}{l}\text { De seis a ocho } \\
\text { meses }\end{array}$ & Certeza total & $\begin{array}{c}\text { 90\% a 75\% de la población } \\
\text { está afectada. }\end{array}$ \\
\hline Alto (4 puntos) & $\begin{array}{l}\text { Dos veces por } \\
\text { año }\end{array}$ & $\begin{array}{c}\text { Cuatro a cinco } \\
\text { meses }\end{array}$ & Muy probable & $\begin{array}{c}26 \% \text { a } 49 \% \text { de la población } \\
\text { está afectada }\end{array}$ \\
\hline Medio (3 puntos) & $\begin{array}{l}\text { Una vez cada } \\
\text { dos años }\end{array}$ & Dos a tres meses & Incierto & $\begin{array}{c}10 \% \text { a } 25 \% \text { de la población } \\
\text { está afectada }\end{array}$ \\
\hline Bajo (2 puntos) & $\begin{array}{l}\text { Una vez cada } \\
\text { cinco años }\end{array}$ & Menos de un mes & Poco probable & $\begin{array}{c}5 \% \text { a 9\% de la población está } \\
\text { afectada }\end{array}$ \\
\hline $\begin{array}{l}\text { Muy bajo (1 } \\
\text { punto) }\end{array}$ & $\begin{array}{l}\text { Una vez cada } \\
\text { diez años }\end{array}$ & $\begin{array}{l}\text { Hasta una } \\
\text { semana }\end{array}$ & $\begin{array}{l}\text { Quizás nunca } \\
\text { ocurra }\end{array}$ & $\begin{array}{l}\text { Menos de un } 4 \% \text { de la } \\
\text { población está afectada. }\end{array}$ \\
\hline
\end{tabular}

Fuente: matriz adaptada de Municipalidad de Filadelfia, 2017. 


\subsubsection{Criterios de evaluación de vulnerabilidad local}

Para la evaluación de vulnerabilidad se partirá desde su definición, entendiéndose como grado en que un sistema natural o social es capaz o incapaz de afrontar los efectos negativos del cambio climático, incluso la variabilidad climática y los episodios extremos. Los rangos de evaluación serán: la exposición que hace referencia a las decisiones y prácticas que ubican a las personas, infraestructuras o actividades económicas en zona de influencia de una amenaza; la fragilidad como la condición de desventaja relativa de personas, infraestructura o actividades económicas frente a una amenaza; y la resiliencia como la capacidad de recuperación frente a la ocurrencia climática. En la Tabla 7 se presenta la matriz con los criterios de evaluación de la vulnerabilidad.

Tabla 7. Criterios de evaluación de vulnerabilidad local para la gestión de cambio climático.

\begin{tabular}{|c|c|c|c|}
\hline $\begin{array}{l}\text { Rangos de } \\
\text { evaluación }\end{array}$ & Exposición & Fragilidad & Resiliencia \\
\hline $\begin{array}{l}\text { Muy alto (5 } \\
\text { puntos) }\end{array}$ & $\begin{array}{l}71 \% \text { al } \\
100 \% \text { de la } \\
\text { población }\end{array}$ & $\begin{array}{l}\text { Muy baja resistencia } \\
\text { ante la amenaza. Bajo } \\
\text { acceso a servicios } \\
\text { básicos y tecnología. }\end{array}$ & $\begin{array}{l}\text { Muy alta. Tiene todas las capacidades naturales, } \\
\text { sociales, económicas, institucionales y tecnológicas } \\
\text { para recuperarse y adaptarse. }\end{array}$ \\
\hline $\begin{array}{l}\text { Alto (4 } \\
\text { puntos) }\end{array}$ & $\begin{array}{l}51 \% \text { a } 70 \% \text { de } \\
\text { la población }\end{array}$ & $\begin{array}{l}\text { Baja resistencia. 60\% } \\
\text { tienen limitaciones } \\
\text { de infraestructura y } \\
\text { tecnología para resistir. }\end{array}$ & $\begin{array}{l}\text { Alta. Tiene capacidades naturales, sociales, } \\
\text { económicas mínimas para recuperarse y adaptarse. }\end{array}$ \\
\hline $\begin{array}{l}\text { Medio (3 } \\
\text { puntos) }\end{array}$ & $\begin{array}{l}26 \% \text { a } \\
50 \% \text { de la } \\
\text { población }\end{array}$ & $\begin{array}{l}40 \% \text { de la población } \\
\text { tienen limitaciones }\end{array}$ & Cuenta con algunas capacidades para recuperarse. \\
\hline $\begin{array}{l}\text { Bajo (2 } \\
\text { puntos) }\end{array}$ & $\begin{array}{l}10 \% \text { a } 25 \% \text { de } \\
\text { la población }\end{array}$ & $\begin{array}{l}\text { El 25\% de la población } \\
\text { tiene limitaciones }\end{array}$ & No tiene capacidades para recuperarse \\
\hline $\begin{array}{l}\text { Muy bajo ( } 1 \\
\text { punto) }\end{array}$ & $\begin{array}{l}\text { Menos del } \\
10 \% \text { de la } \\
\text { población }\end{array}$ & $\begin{array}{l}\text { La mayor parte de la } \\
\text { población tiene buena } \\
\text { condición económica } \\
\text { y tiener mejores } \\
\text { condiciones sociales. }\end{array}$ & $\begin{array}{l}\text { Ausencia total de capacidades y medios para } \\
\text { enfrentar la variabilidad climática }\end{array}$ \\
\hline
\end{tabular}

Fuente: matriz adaptada de Municipalidad de Filadelfia, 2017.

\subsubsection{Evaluación de amenaza y vulnerabilidad y nivel de riesgo}

Para evaluar la amenaza y vulnerabilidad se sumará el puntaje de los criterios correspondientes y para el nivel de riesgo estos dos valores también se sumarán, teniendo en cuenta que de 1 a 7 es muy bajo, de 8 a 14 es bajo, de 15 a 21 es medio, de 22 a 28 es alto y de 29 a 35 es muy alto. En la Tabla 7, se presenta el formato, que puede ser modificado según sea el caso, para evaluar el nivel del riesgo en cada una de las tres zonas nombradas anteriormente.

Tabla 8. Matriz de evaluación de riesgos climáticos locales.

\begin{tabular}{|c|c|c|c|c|c|c|c|c|c|c|c|}
\hline \multirow{2}{*}{$\begin{array}{l}\text { Eventos/ } \\
\text { Efecto }\end{array}$} & \multirow{2}{*}{ Sistemas afectados } & \multicolumn{5}{|c|}{ Amenaza } & \multicolumn{4}{|c|}{ Vulnerabilidad } & \multirow{2}{*}{ NR } \\
\hline & & $\mathrm{F}$ & D & $\mathrm{P}$ & $\mathrm{C}$ & E & E & F & $\mathrm{R}$ & $\mathrm{E}$ & \\
\hline \multirow{8}{*}{ Sequía } & $\begin{array}{l}\text { Movilidad vías y } \\
\text { transporte }\end{array}$ & 5 & 4 & 2 & 3 & 14 & 4 & 3 & 5 & 12 & ALTO \\
\hline & $\begin{array}{c}\text { Equipamientos } \\
\text { colectivos }\end{array}$ & & & & & & & & & & \\
\hline & Espacio publico & & & & & & & & & & \\
\hline & $\begin{array}{c}\text { Sistemas } \\
\text { productivos }\end{array}$ & & & & & & & & & & \\
\hline & $\begin{array}{c}\text { Estructura } \\
\text { ecológica principal } \\
\end{array}$ & & & & & & & & & & \\
\hline & $\begin{array}{c}\text { Servicios públicos } \\
\text { domiciliarios }\end{array}$ & & & & & & & & & & \\
\hline & Vivienda y hábitat & & & & & & & & & & \\
\hline & $\begin{array}{c}\text { Patrimonio } \\
\text { cultural }\end{array}$ & & & & & & & & & & \\
\hline
\end{tabular}




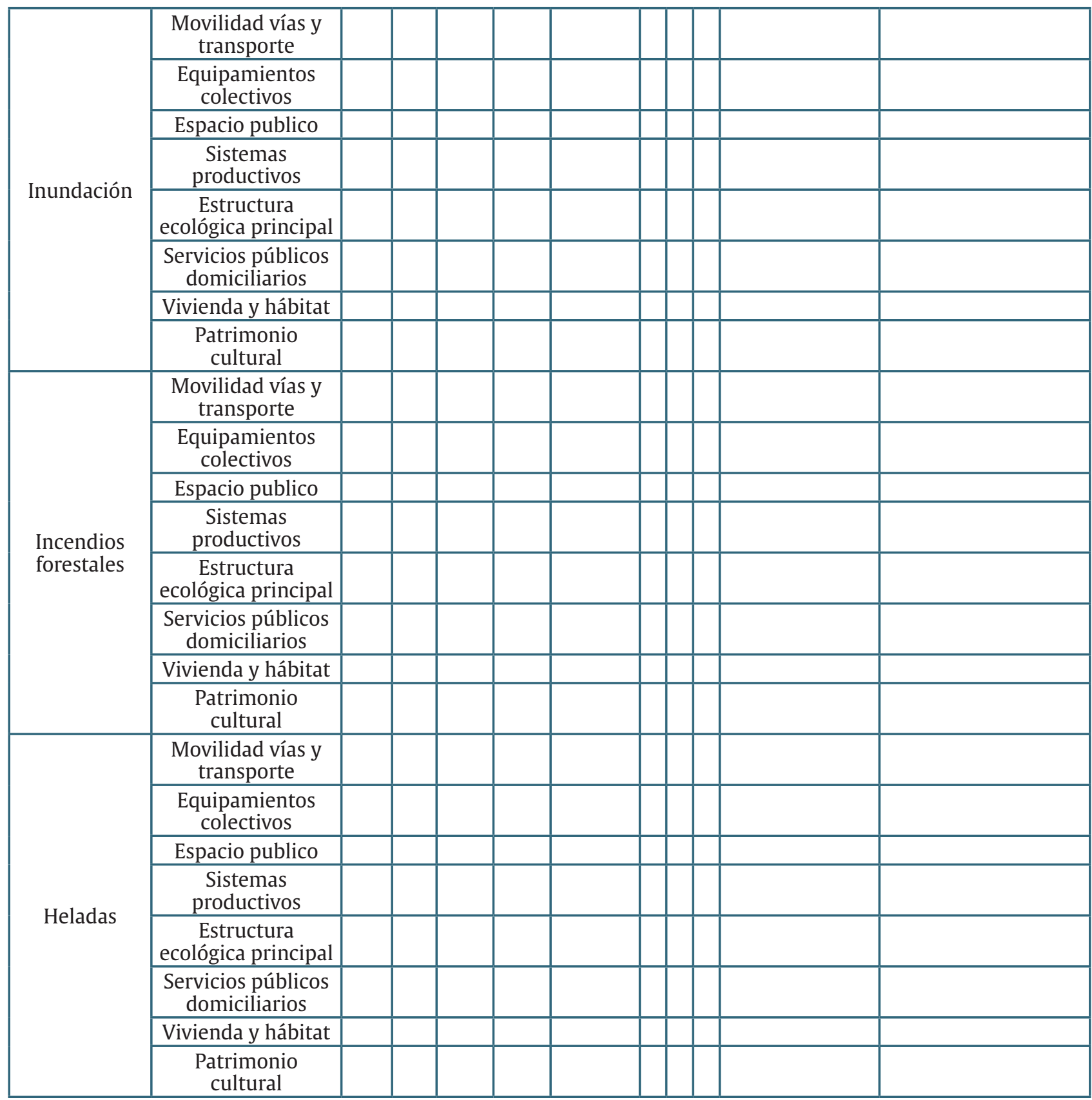

Fuente: adaptado de Municipalidad de Filadelfia, 2017.

\subsection{Etapa 2: Formulación de estrategias}

En esta sección el gestor de cambio climático formulará las estrategias frente al cambio climático, que en esencia corresponden a proyectos o programas que deben implementarse para avanzar en el cumplimiento de las metas nacionales del país, encaminadas a la adaptación y mitigación de los riesgos climáticos.

Las acciones se formularán desde las líneas estratégicas establecidas en la PNCC, que corresponden al desarrollo territorial, desarrollo minero energético (si aplica), desarrollo de infraestructura estratégica, manejo y conservación de los ecosistemas y sus servicios ecosistémicos, todos enfocados al desarrollo bajo en carbono y resiliente al clima.

Por lo anterior se propone una matriz, donde el gestor de cambio climático podrá diligenciar el nombre de la estrategia; una breve descripción de las implicaciones y aspectos para tener en cuenta en su desarrollo; la escala; la zona que comprende; la localización de la acción, que puede ser por veredas o referentes geográficos y la temporalidad. A continuación, se presentan una serie de tablas en las que se explican cada uno de estos ítems. 
En cuanto a la escala, esta puede ser: veredal, municipal, regional o multiescalar.

Tabla 9. Descripción de las escalas de implementación de las estrategias.

\begin{tabular}{|c|l|}
\hline Escala & \multicolumn{1}{c|}{ Descripción } \\
\hline Veredal & Se refiere a acciones que deben ser implementadas únicamente en zonas rurales \\
\hline Municipal & Se refiere a acciones que deben ser implementadas únicamente en zonas urbanas \\
\hline Regional & $\begin{array}{l}\text { Se refiere a acciones que deben ser implementadas y/o concebidas desde una } \\
\text { perspectiva regional (Supramunicipal) }\end{array}$ \\
\hline Multiescalar & $\begin{array}{l}\text { Se refiere a acciones que deben ser implementadas y/o } \\
\text { concebidas a diferentes escalas, desde lo local (Veredal y Municipal) hasta lo } \\
\text { Regional (Supramunicipal) }\end{array}$ \\
\hline
\end{tabular}

Fuente: adaptado de Corporación Autónoma Regional de Risaralda; Gobernación de Risaralda, 2013.

En cuanto a la zona, esta puede ser: rural, urbana o suburbana.

Tabla 10. Descripción de la zona de las estrategias.

\begin{tabular}{|c|l|}
\hline Zona & \multicolumn{1}{c|}{ Descripción } \\
\hline Rural & Se refiere a acciones que deben ser implementadas únicamente en zonas rurales \\
\hline Urbana & Se refiere a acciones que deben ser implementadas únicamente en zonas urbanas \\
\hline Suburbana & $\begin{array}{l}\text { Donde se mezclan las zonas y formas de vida del campo y la ciudad, garantizando el } \\
\text { autoabastecimiento en servicios públicos. }\end{array}$ \\
\hline
\end{tabular}

Fuente: adaptado de Corporación Autónoma Regional de Risaralda; Gobernación de Risaralda, 2013.

Por su parte, la localización de la estrategia se refiere al "lugar" de implementación, este puede ser por vereda, por cuenca hidrográfica o por referentes geográficos, entre otros.

En cuanto a la temporalidad, esta puede ser; en el corto plazo, en el mediano plazo, en el largo plazo o muy largo plazo.

Tabla 11. Descripción de las temporalidades de implementación estrategias.

\begin{tabular}{|c|l|}
\hline Temporalidad & \multicolumn{1}{|c|}{ Descripción } \\
\hline $\begin{array}{c}\text { Corto plazo } \\
\text { (año uno, dos tres, cuatro y cinco) }\end{array}$ & $\begin{array}{l}\text { Estrategias que deberían ser implementadas } \\
\text { en un horizonte no superior a cinco años }\end{array}$ \\
\hline $\begin{array}{c}\text { Mediano plazo } \\
\text { (año seis, siete, ocho, nueve y diez) }\end{array}$ & $\begin{array}{l}\text { Estrategias que podrían ser implementadas } \\
\text { después del corto plazo, no superior a diez años. }\end{array}$ \\
\hline $\begin{array}{c}\text { Largo plazo } \\
\text { (año 11, 12,13,14 y 15) }\end{array}$ & $\begin{array}{l}\text { Estrategias que podrían ser implementadas } \\
\text { despuésdelmedianoplazo,nosuperiora15años. }\end{array}$ \\
\hline Muy largo plazo & $\begin{array}{l}\text { Estrategias que podrían ser implementadas } \\
\text { después del largo plazo, no superior a 20 años. }\end{array}$ \\
\hline
\end{tabular}

Fuente: adaptado de Corporación Autónoma Regional de Risaralda; Gobernación de Risaralda, 2013.

A continuación, se presenta el formato con un ejemplo para que el gestor se pueda guiar en la formulación de las estrategias encaminadas a la mitigación y adaptación del territorio. 
Tabla 12. Matriz de estrategias para la gestión del cambio climático.

\begin{tabular}{|c|c|c|c|c|c|}
\hline \multicolumn{6}{|c|}{ Línea estratégica de la PNCC: } \\
\hline Número & Estrategia & Escala & Zona & Localización & Temporalidad \\
\hline \multirow{4}{*}{1} & $\begin{array}{c}\text { Incorporación } \\
\text { de } \\
\text { prácticas de } \\
\text { conservación } \\
\text { en los } \\
\text { sistemas } \\
\text { productivos } \\
\text { rurales del } \\
\text { municipio }\end{array}$ & Municipal & Rural & Veredas & Corto plazo \\
& $\begin{array}{l}\text { Tiene que ver con la necesidad de continuar con la reconversión socioambiental } \\
\text { de los "actuales" sistemas productivos agropecuarios y forestales del municipio } \\
\text { con el fin de hacerlos más sostenibles y competitivos en el largo plazo. }\end{array}$ \\
\hline
\end{tabular}

Fuente: adaptado de Corporación Autónoma Regional de Risaralda; Gobernación de Risaralda, 2013.

\subsection{Etapa 3: Medios de implementación}

En esta etapa se establecerán los medios de implementación por línea estratégica de la PNCC, diligenciando cada una de las estrategias formuladas anteriormente. En la Tabla 13 se presenta el formato de matriz para cada una de las líneas estratégicas, donde se diligenciará: el nombre de la estrategia; el nombre de el o los actores que implementarán la medida; el rol que cumplen, que puede ser operativo, financiero o técnico; la descripción de su función para la estrategia; si aplica, las políticas municipales que debiesen ser implementadas y/o modificadas para efectuar la estrategia; la información necesaria para implementar esta estrategia; la tecnología o infraestructura que se requiera; y la capacidad técnica necesaria, que puede ser de tipo profesional, técnico o tecnológico.

Tabla 13. Matriz medios de implementación de estrategias climáticas locales.

\begin{tabular}{|c|c|c|}
\hline \multicolumn{2}{|c|}{ Línea estratégica de la PNCC: Desarrollo bajo en carbono } \\
\hline \multicolumn{2}{|c|}{ Estrategia: Formulación del plan de acción por el clima } \\
\hline Nombre del actor & Rol Técnico & $\begin{array}{c}\text { Capacitar a los funcionarios en } \\
\text { procesos de gestión del cambio } \\
\text { climático }\end{array}$ \\
\hline $\begin{array}{l}\text { Políticas municipales que debiesen se implementadas y/o } \\
\text { modificadas para aplicar la estrategia }\end{array}$ & Ninguna \\
\hline $\begin{array}{l}\text { Información necesaria para la implementación de la medida } \\
\text { Fuente de financiamiento para la medida }\end{array}$ & $\begin{array}{c}\text { Revisión de conocimientos en los } \\
\text { funcionarios }\end{array}$ \\
\hline Tecnología o infraestructura para la implementación de la media & Herramientas ofimáticas \\
\hline $\begin{array}{l}\text { Capacidades técnicas necesarias para la implementación de la } \\
\text { medida }\end{array}$ & $\begin{array}{c}\text { Nivel de estudios tecnológicos o } \\
\text { profesional }\end{array}$ \\
\hline
\end{tabular}

Fuente: adaptado de Reveco, Harris y Britt, 2015.

\subsection{Etapa 4: Seguimiento de la gestión}

Anualmente, se realizará un seguimiento de la gestión del cambio climático de las tres etapas anteriores, lo que implica la revisión de inversión anual identificada del PPI realizado en la primera etapa, para la cual se utilizará el Plan Operativo Anual de Inversiones (POIAI), instrumento mediante el cual se concretan las inversiones anuales del PPI, así como el seguimiento del progreso de las estrategias formuladas en la segunda etapa, y los medios de implementación que contribuyen a la gestión del cambio climático en el territorio identificados en la tercera etapa.

En la Tabla 14 se presenta un formato de matriz para el seguimiento anual del POAI, donde se indica el sector de desarrollo y programas y/o proyectos acordes con los identificados en la primera etapa, identificando la inversión anual, así como el porcentaje de avance. 
Tabla 14. Matriz de seguimiento anual gestión de cambio climático desde el POAI.

\begin{tabular}{|c|c|c|c|c|}
\hline $\begin{array}{c}\text { Sector de } \\
\text { desarrollo }\end{array}$ & $\begin{array}{c}\text { Programa } \\
\text { y/o } \\
\text { proyecto }\end{array}$ & $\begin{array}{c}\text { Inversión } \\
\text { anual } \\
\text { (Pesos) }\end{array}$ & $\begin{array}{c}\text { Progreso } \\
\text { de la } \\
\text { inversión } \\
\text { (Pesos) }\end{array}$ & $\begin{array}{c}\text { Porcentaje } \\
\text { de progreso } \\
(\%)\end{array}$ \\
\hline $\begin{array}{c}\text { Cambio } \\
\text { climático }\end{array}$ & $\begin{array}{c}\text { Acción por } \\
\text { el clima }\end{array}$ & $\$ 3.000 .000$ & $\$ 100.000$ & $3,3 \%$ \\
\hline
\end{tabular}

Fuente: elaboración propia.

En la Tabla 15 se presenta el formato de matriz para el seguimiento anual de las estrategias propuestas en la segunda etapa, donde determinará la unidad de medida, que puede ser en pesos, informes, eventos, entre otras; la meta anual según la unidad de medida escogida; el progreso anual de la meta; y finalmente el porcentaje de progreso que se cumplió para la implementación de la estrategia al final del año.

Tabla 15. Matriz anual se seguimiento de estrategia de gestión de cambio climático local.

\begin{tabular}{|c|c|c|c|c|}
\hline \multicolumn{4}{|c|}{ Línea estratégica de la PNCC: Desarrollo bajo en carbono } \\
\hline Estrategia & $\begin{array}{c}\text { Unidad de } \\
\text { medida }\end{array}$ & $\begin{array}{c}\text { Meta } \\
\text { anual }\end{array}$ & $\begin{array}{c}\text { Progreso } \\
\text { de la } \\
\text { meta }\end{array}$ & $\begin{array}{c}\text { Porcentaje } \\
\text { de progreso } \\
(\%)\end{array}$ \\
\hline $\begin{array}{c}\text { Talleres } \\
\text { comunitarios } \\
\text { en materia } \\
\text { de cambio } \\
\text { climático }\end{array}$ & $\begin{array}{c}\text { Numero } \\
\text { de talleres } \\
\text { al año }\end{array}$ & 4 & 1 & $25 \%$ \\
\hline
\end{tabular}

Fuente: elaboración propia.

\section{Análisis de resultados}

En este trabajo se identificaron cuatro elementos metodológicos principales: planificación y diagnóstico, formulación de estrategias, medios de implementación y seguimiento de la gestión; que en su orden respectivo conforman una guía que contribuye a la optimización de la gestión del cambio climático a nivel local en Colombia. Desde la revisión documental de instrumentos a nivel nacional, se evidenció que los elementos metodológicos principales identificados están planteados desde lineamientos propuestos en documentos de orden nacional, como lo es el documento "Lineamientos de Política de Cambio Climático" propuesto por el Ministerio de Medio Ambiente y Desarrollo Sostenible (MADS), unos de los primeros documentos que dio las bases de política pública en materia de cambio climático, tomando como referente las normativas a nivel internacional como lo es la Convención Marco de las Naciones Unidas sobre el Cambio Climático (CMNUCC), identificando los principales impactos del cambio climático en Colombia y su implicaciones con el CMNUCC y el Protocolo de Kioto para Colombia (Departamento Nacional de Planeación; Ministerio de Medio Ambiente y Desarrollo Sostenible; Instituto de Hidrología y Meteorología, 2002).

Para el primer elemento metodológico, correspondiente a planificación y diagnóstico, se propone una identificación de la gestión de cambio climático en otros instrumentos de planificación a nivel local. Los entes territoriales a nivel local cuentan con varios instrumentos de planificación en los cuales se plantean acciones desde sus ejes programáticos, por lo cual es importante identificar si existen planes, programas o presupuesto que pueden ser clasificados como estrategias que aporten al proceso de gestión del cambio climático, como evitar duplicidad de competencias y aumentar la eficacia de procesos.

Posteriormente, la formulación del perfil de gestión climática local se plantea a partir de la importancia de conocer el estado de la gestión del cambio climático en el territorio, conocer los recursos, infraestructura y recurso humano con el que se cuenta para este proceso.

Aunque esta guía va dirigida hacia la gestión del cambio climático, es importante conocer los riesgos para poder gestionar los procesos a nivel local, por lo cual se estableció dentro de este elemento el análisis de riesgos climáticos locales, que permite identificar las amenazas, la vulnerabilidad y el nivel del riesgo al que se expone el territorio.

Otros dos elementos metodológicos identificados fueron la formulación de estrategias y medios de implementación, que trabajan en sinergia para la optimización de la gestión pues permite conocer las acciones que se van a realizar desde el análisis de riesgos, y los actores que participaran en la ejecución de 
la gestión.

El último elemento es el seguimiento de la gestión, pues el cambio climático es un fenómeno que cambia con el tiempo y requiere de constantes actualizaciones desde las bases científicas y la gestión, pues los fenómenos pueden cambiar o los riesgos se pueden agudizar, y desde la gestión los instrumentos de planificación cada cierto periodo de tiempo se actualiza, por lo tanto, el seguimiento es primordial para que la gestión del cambio climático mantenga su óptimo desarrollo.

Con el desarrollo de estos elementos, se diseñó la guía conformada por cuatro etapas que mantienen un orden secuencial comenzando desde la revisión y análisis de la gestión del cambio climático, y finalizando en el seguimiento desde las estrategias y medios de implementación formulados que permitan la optimización de la gestión del cambio climático a nivel local en Colombia.

Inmersas en cada una de las etapas se encuentran los elementos que permiten optimizar la gestión, a través de matrices que faciliten la visualización e implementación de medidas de gestión del cambio climático, con lo cual se podrá evitar duplicidades de competencias y de identificación de instrumentos territoriales, las cuales también contemplan información física y sociodemográfica del municipio y la inclusión de minorías étnicas.

Esta guía está compuesta por solo cuatro etapas, ofrece a las autoridades locales o tomadores de decisiones una guía comprensible y en línea con parámetros establecidos por autoridades nacionales en materia de cambio climático, como los son las "Orientaciones para la inclusión del cambio climático en los planes de ordenamiento territorial (POT, PBOT, EOT)" (Corporacion Autónoma Regional, 2018) o "Consideraciones de cambio climático para el ordenamiento territorial" (Programa de las Naciones Unidas para el Desarrollo; Ministerio de Ambiente y Desarrollo Sostenible; Gobierno de Colombia, 2018), logrando con esta guía identificar elementos principales que tengan una relación armónica entre territorio y cambio climático.

Estos elementos se proponen desde una revisión de instrumentos locales nacionales e internacionales hasta el año 2020, proponiendo una guía que pueda ser implementada a nivel local, sin embargo, es necesario indagar constantemente la bibliografía, pues como el cambio climático no es un fenómeno estable sus bases conceptuales y científicas cambian para acoplarse a la realidad. Así mismo, estos elementos se proponen desde una perspectiva conceptual, por lo cual es necesario profundizar en las técnicas científicas para los análisis de riesgos climáticos, tener en cuenta los escenarios de cambio climático para la formulación e implementación de estrategias que contribuyan a la mitigación y adaptación al cambio climático, y mejorar la gestión del cambio climático a nivel local.

\section{Conclusiones}

Los elementos metodológicos principales para la gestión del cambio climático a nivel local en Colombia son los seis siguientes: planificación y diagnóstico, formulación de estrategias, medios de implementación y seguimiento de la gestión.

Esta guía que se propone establecer los elementos metodológicos principales que optimicen la gestión local de cambio climático, responde a un carácter integral de la gestión identificando acciones desde otros instrumentos de planificación, incluyendo las acciones que abordan el cambio climático por medio de un inventario de información que se conoce en relación con el clima, sus impactos, actores involucrados, permitiendo orientar a los tomadores de decisiones locales, en el uso y manejo del territorio con miras a afrontar los efectos del cambio y la variabilidad climática en un horizonte de 30 años.

El desarrollo de este trabajo contribuye al proceso de investigación que permite conocer conceptos básicos comprendiendo las causas y consecuencias de las interacciones climáticas con las actividades antrópicas desde la gestión del cambio climático, y haciendo un llamado a el compromiso de hacer que este tema cobre importancia en la comunidad y lograr acciones en todo nivel que contribuirán globalmente a la mitigación y adaptación ante el cambio climático.

Este trabajo es un primer esfuerzo en plantear los elementos metodológicos a nivel local en Colombia, que toma sus bases conceptuales de gestión del cambio climático documentadas desde la Política Nacional de Cambio Climático y la Ley 1931 de 2018 de la República de Colombia.

Este documento, además de proporcionar una documentación, revisión y comparación de bibliografía internacional y colombiana, se destaca la convergencia entre el ordenamiento territorial y el cambio climático desde una agenda de desarrollo integral y no como un problema ambiental. 


\section{Referencias bibliográficas}

Congreso de la República de Colombia. (2018). Ley 1931. 1-19. Recuperado de: http://extwprlegs1.fao.org/ docs/pdf/col191539.pdf

Convención Marco de las Naciones Unidas sobre el Cambio Climático (CMNUCC). (1997). Protocolo de Kioto. Convención Marco de las Naciones Unidas sobre el Cambio Climático, 61702, 25. Recuperado de: https://unfccc.int/resource/docs/convkp/kpspan.pdf

Corporacion Autónoma Regional de Colombia. (2018). Orientaciones para la inclusión del cambio climático en los planes de ordenamient territorial (POT, PBOT, EOT). Colombia. Recuperado de: https://www. car.gov.co/uploads/files/5b070c9370ad3.pdf

Corporación Autónoma Regional de Risaralda; Gobernación de Risaralda. (2013). Plan Departamental de Gestion de Cambio Climático - Risaralda. 101. Colombia. Recuperado de: http://www.inci.gov.co/ observatorio-social/informacion-territorial/san-andres-y-providencia

Christlieb, F. F. (2012). Corografía y Escala Local. Enfoques desde la Geografía. Morelia, Michoacán, México: Universidad Nacional Autónoma de México. Recuperado de: http://www.igg.unam.mx/sigg/ cultural/vista/archivos/publicacion/1449478928corografiaayaescalaalocalapdf.pdf

Departamento Nacional de Planeación; Ministerio de Medio Ambiente y Desarrollo Sostenible; Instituto de Hidrología y Meteorología de Colombia. (2002). Lineamientos de la Política de Cambio Climático. Colombia. Recuperado de: https://www.preventionweb.net/ files/21403_15719lineamientospoliticanacionalca.pdf

Departamento Nacional de Planeación de Colombia. (2011). Conpes 3700 de 2011. Estrategia insititucional para la articulación y acciones en materia de cambio climático en Colombia. 75. Colombia. Recuperado de: https://colaboracion.dnp.gov.co/CDT/Conpes/Econ\%C3\%B3micos/3700.pdf

Grupo Intergubernamental de Expertos sobre Cambio Climático (IPCC). (2013). Glosario. Recuperado de: https://www.ipcc.ch/site/assets/uploads/2018/08/WGI_AR5_glossary_ES.pdf

Ministerio de Medio Ambiente y Desarrollo Sostenible de Colombia. (2016). Política nacional de cambio climático. Colombia. Recuperado de: https://www.minambiente.gov.co/images/cambioclimatico/ pdf/Politica_Nacional_de_Cambio_Climatico_-_PNCC_/PNCC_Politicas_Publicas_LIBRO_Final_ Web_01.pdf

Ministerio de Medio Ambiente y Desarrollo Sostenible de Colombia. (2017). Politica Nacional de Cambio Climático. Colombia. Recuperado de: http://www.minambiente.gov.co/images/cambioclimatico/ pdf/Politica_Nacional_de_Cambio_Climatico_-_PNCC_/PNCC_Politicas_Publicas_LIBRO_Final_ Web_01.pdf

Ministerio de Medio Ambiente y Desarrollo Sostenible de Colombia. (2020). Aproximación a territorio: Planes Integrales de Gestión de Cambio Climático Territoriales - PIGCCT | Ministerio de Ambiente y Desarrollo Sostenible. Colombia. Recuperado de: https://www.minambiente.gov.co/index.php/ gestion-territorial-de-cambio-climatico/aproximacion-a-territorio-planes-territoriales-decambio-climatico

Municipalidad de Filadelfia. (2017). Plan municipal de adaptación al cambio climático de Filadelfia 20172022. 76. Paraguay. Recuperado de: http://www.granchacoproadapt.org/portal/documentos/ PlanFiladelfia.pdf

Municipalidad de Vitacura, Chile. (2019). Plan local de cambio climático - Vitacura. Chile. Recuperado de: https://www.vitacura.cl/medio-ambiente/cambio-climatico.html

Programa de las Naciones Unidas para el Desarrollo; Ministerio de Ambiente y Desarrollo Sostenible; Gobierno de Colombia. (2018). Consideraciones de cambio climático para el ordenamiento territorial. Colombia. Recuperdado de https://www.minambiente.gov.co/images/cambioclimatico/pdf/desarrollo_y_ planificacion/Consideraciones_de_Cambio_Climatico_para_el_Ordenamiento_Territorial_VF.pdf

Programa de las Naciones Unidas para el Desarrollo; Ministerio de Ambiente y Desarrollo Sostenible; Instituto de Hidrología y Meteorología de Colombia. (2010). Segunda Comunicación Nacional de Colombia. 17-43. Colombia. Recuperado de: http://documentacion.ideam.gov.co/openbiblio/ bvirtual/021658/2Comunicacion/IDEAMTOMOIIPreliminares.pdf

Reveco, C., Harris, J., y Britt, J. (2015). Herramienta 8: Academias de Cambio Climático: Planificar la adaptación en el ámbito local. Serie de Estudios Temáticos EUROCLIMA de la Comisión Europea (Vol. 8). doi: https:/l doi.org/978-956-9708-02-2 